\title{
L'Énonciation culturelle chez Panaït Istrati et Patrick Chamoiseau : traduction ou trahison?
}

\author{
MARIANA C. IONESCU \\ Huron University College
}

Résumé

S'inspirant des idées avancées par Homi Bhabha au sujet de la difficulté de représenter une tradition culturelle dans une autre langue, cet article aborde la question de la traductibilité d'un dit profondément ancré dans une culture essentiellement orale. Chez Panaït Istrati, écrivain français d'origine roumaine, aussi bien que chez le Martiniquais Patrick Chamoiseau, récipiendaire du prix Goncourt, la mise en écrit des paroles d'un conteur est-elle donc un acte de traduction ou une trahison de la diction originelle? Dans les deux cas, on constatera que l'écriture tisse un espace linguistique hybride portant les traces de l'oral qui l'in-forme.

À première vue, les Récits d'Adrien Zograffi de Panaït Istrati, publiés à Paris dans les années 20, n'ont rien en commun avec Solibo Magnifique du Martiniquais Patrick Chamoiseau, paru chez Gallimard en 1988. Cependant, une même tension les sous-tend, tension ayant comme point d'origine l'acte d'énonciation qui met en présence deux langues : d'un côté le roumain et, respectivement le créole, porteurs de richesses culturelles hybrides, d'un autre côté le français, langue d'écriture de ces deux auteurs.

Prenant comme point de départ les idées avancées par Homi Bhabha au sujet de la difficulté de représenter une tradition culturelle dans une autre langue, nous aborderons la question de la traductibilité d'un dit profondément ancré dans une langue et dans un système de référence fournis par une culture particulière. Si toute culture est le produit d'un discours, la mise en écrit des paroles d'un conteur représente-t-elle un acte de traduction ou une trahison de la diction originelle? Selon Bhabha, I'acte de I'énonciation culturelle interfère avec l'écrit, donnant naissance à un tiers espace, celui de la traduction culturelle, espace frontalier à partir duquel se façonne le pacte ambivalent de l'interprétation culturelle (Bhabha 1994). Aussi, le sujet de l'énonciation, en l'occurrence le conteur fictionnalisé, entre-t-il en dialogue avec le sujet de l'énoncé, que ce soit le marqueur de paroles ${ }^{1}$ Chamoiseau, ou le jeune Adrien, porte-parole d'Istrati. À travers ce dialogue se tisse un espace de création linguistique hybride, lieu d'aliénation de la parole, lieu de scission du sujet énonciateur, mais aussi lieu d'enrichissement de l'écrit au contact avec une tradition culturelle devenue forcément littérature.

\section{Panaït Istrati}

Afin de mieux comprendre les circonstances qui ont favorisé le choix du français comme langue d'écriture d'Istrati, quelques brefs repères biobibliographiques seront utiles aux lecteurs qui ignorent le parcours sinueux de cet auteur autodidacte.

Né en 1884 à Braïla, ville portuaire cosmopolite de l'est de la Roumanie, d'un père grec et d'une mère roumaine, Panaït Istrati passe sa petite enfance chez la famille de sa mère, dans le village de Baldovineşti. L'enfant y sera fasciné par les histoires ayant comme personnages privilégiés les haïdoucs, hors-la-loi légendaires du folklore roumain². Après l'école 
primaire, le jeune Panaït arrête ses études et exerce divers métiers dans sa ville natale. Plus tard, entré en contact avec le mouvement socialiste, il commence à publier des articles dans le journal La Roumanie ouvrière, où il exprime sa solidarité avec tous les déshérités du monde.

À part la passion constante pour la lecture, Istrati manifeste très tôt un désir inassouvi de voyage et d'amitié. Sa quête fiévreuse d'âmes passionnées et de contrées nouvelles le pousse à parcourir l'Égypte, la Grèce, l'Italie et les pays du Proche-Orient, seul ou en compagnie d'amis inoubliables entrés plus tard dans plusieurs de ses récits. Au cours de ses nombreuses traversées de l'Orient, une force inexorable le pousse vers l'Occident, notamment vers la France, où il arrivera en 1913. La première guerre mondiale surprend Istrati en Suisse où, atteint de tuberculose, il est hospitalisé plusieurs fois. C'est là qu'il se met à apprendre le français, ayant comme maîtres les livres de Voltaire, de Rousseau et de Fénelon. Quelques années plus tard, après une tentative de suicide à Nice, il rencontrera son mentor, Romain Rolland, le premier à avoir eu l'intuition de son don de conteur. Celui-ci ne cessera d'encourager le jeune Roumain de mettre sur papier les histoires de son terroir, ainsi que celles issues de son long périple à travers l'Orient.

Bien que conseillé dès le début par R. Rolland de ne pas s'aventurer sur le terrain du roman-fleuve, Istrati ne renonce pas à son idée initiale de créer un cycle romanesque ayant comme protagoniste Adrien Zograffi. Les Récits d'Adrien Zograffi suivent les pas de son alter ego diégétique dans le temps (de l'enfance à l'adolescence) et dans l'espace (de l'Orient à I'Occident). Dans les quatre premiers volumes, Kyra Kyralina, Oncle Anghel, Présentation des haïdoucs et Domnitza de Snagov, le jeune Adrien assume le plus souvent le rôle de narrataire, prenant place quelquefois parmi d'autres narrataires fictifs assis autour d'un conteur pour écouter des histoires haïdouques ${ }^{3}$. L'auteur lui-même le présente comme tel dans le discours préfaciel de plusieurs de ses récits. Ainsi, dans la préface au roman La Maison Thüringer (1932), Istrati évoque-t-il métaphoriquement le parcours de son écriture des premiers récits, où se font entendre les sons « d'une flûte aux sons enchanteurs », vers ses derniers romans, où la flûte cède graduellement la place à la plume de l'écrivain. C'est ce cycle de la flûte 4 qui présente le plus d'intérêt pour la question de l'énonciation culturelle.

\section{La langue d'écriture d'Istrati}

À l'apparition du premier volume d'Istrati, Kyra Kyralina, en 1924, la critique française se montre élogieuse. On en apprécie la simplicité et la nouveauté, l'impression de fraîcheur et de primitivisme, et surtout le don de conteur de l'écrivain : « Il est conteur-né », écrit Romain Rolland dans la préface à ce récit, « un conteur d'Orient, qui s'enchante et s'émeut de ses propres récits, et si bien s'y laisse prendre qu'une fois l'histoire commencée, nul ne sait, ni lui-même, si elle durera une heure, ou bien mille et une nuit. »

Si les Français accueillent favorablement le début littéraire d'Istrati, les positions adoptées par les critiques roumains d'avant et d'après la guerre se révèlent extrêmement divergentes, surtout à cause des différences entre leur horizon d'attente socioculturel. L'essence des attaques contre les écrits istratiens repose sur l'idée de la mystification des réalités roumaines, que cet écrivain aurait accomplie dans une langue autre que la sienne, raison pour laquelle, de l'avis des

http://www.brocku.ca/cfra/voixplurielles05-02/index.html 
grands critiques Nicolae Iorga ou George Călinescu, il ne pourrait jamais être considéré écrivain roumain. D'autres critiques et écrivains tels Garabet Ibrăileanu, Camil Petrescu ou Alexandru Philippide, tout en appréciant les qualités littéraires des premiers récits d'Istrati, expriment leur regret sur le choix que l'auteur avait fait en matière de langue d'écriture.

Des études de date plus récente, comme celles d'Elisabeth Geblesco, de Zamfir Bălan ou de Cecilia Condei, ont abordé les textes istratiens par le biais de la psychanalyse, de la linguistique ou de la narratologie, sans pour autant avoir tranché sur le statut de cet écrivain. La question épineuse de la langue d'écriture d’Istrati est soulevée également par Alain Schaffner dans un article publié en 2004 dans L'Esprit Créateur : « Le choix du français par Istrati n'est-il pas tout de même une sorte de trahison à l'égard du roumain? » (56) L'auteur de l'article en question amorce une réponse dans laquelle il rapproche Istrati d'un autre grand exilé, Cioran, en concluant qu'une même aliénation provoquée par le changement de langue est évidente dans certaines déclarations des deux écrivains. À ce sujet, il fournit un extrait d'un manuscrit d'Istrati datant de 1921, publié dans Esprit :

Je maudis [...] cette circonstance qui, au lieu de me permettre d'écrire dans ma propre langue, qui m'est familière, $\mathrm{m}$ 'a banni à mille lieues de mon pays et me force de perdre le temps dans des recherches de dictionnaire, pour la stupide raison que dans tel endroit il faut deux l, deux m, deux $n$, et qu'un seul n'est pas suffisant. Mais, au risque de paraître ridicule, je m'accroche à cette langue que j'aime et que je veux connaître. (Istrati 1977 [1921], 378)

\section{L'entre-deux culturel istratien}

Qu'il soit considéré écrivain roumain de langue française ou écrivain français d'origine roumaine, il ne fait aucun doute que cet auteur, à qui le français tendait des pièges à chaque pas, pensait en roumain et écrivait en français. Cependant, en 1933, après avoir buté contre la difficulté de traduire en roumain quelques-uns de ses textes français, Istrati fait cette déclaration pour le moins paradoxale : « Je suis venu dans les lettres françaises avec une âme roumaine, mais je dus lui prêter un masque français. Quand je tentai de rendre à cette âme son visage roumain, je ne le pouvais plus; elle s'était éloignée avec un visage étranger. $\gg^{5}$

Cet aveu soulève, d'un côté, la question de la fidélité de la représentation culturelle dans une langue autre que celle du groupe ethnique représenté par un auteur, et de l'autre côté, celle de l'effet produit par la traduction de la même représentation culturelle dans la langue maternelle de l'auteur en question. Dans ses études sur la langue d'Istrati, Vasile Covaci justifie la présence des expressions et mots roumains par la tentative de l'écrivain de traduire un hypothétique texte roumain qui parfois s'avérait intraduisible. Ce postulat figure également dans les recherches plus récentes de Tamara Ceban qui, lors d'une conférence donnée au mois de mars 2006 à Chişinău, a présenté les champs lexicaux roumains les plus significatifs auxquels Istrati avait recourt faute d'équivalent en français. En poussant cette idée plus loin, nous avancerons I'hypothèse que cette traduction d'une civilisation pour la plupart méconnue en Occident a donné naissance à un troisième espace, véritable entre-deux culturel et linguistique surgi à la suite de l'interférence de l'oral et de l'écrit.

Ceci dit, examinons de plus près les premiers récits d'Istrati qui trahissent davantage l'impact de l'art narratif oral manifesté au niveau de la structure, du contenu, des instances d'énonciation, aussi bien qu'au niveau du vocabulaire. C'est sur ce dernier niveau que porteront nos observations sur l'énonciation culturelle chez Istrati ${ }^{6}$.

http://www.brocku.ca/cfra/voixplurielles05-02/index.html 
D'entrée de jeu nous dirons que le besoin de se faire comprendre par un lecteur français aurait obligé Istrati de limiter au maximum le nombre des mots roumains présents dans ses récits. Or, l'auteur préfère en utiliser plus de 400 , la plupart chargés de connotations multiples dans la tradition culturelle roumaine. A-t-il voulu tout simplement « faire entendre au lecteur les sonorités du roumain », comme le suppose Alain Schaffner? (55) Aurait-il choisi d'émailler ses récits de mots de sa langue maternelle pour les rendre plus exotiques? Les mots roumains trahissent-ils le « désir phantasmatique primordial » de l'auteur d'infuser la langue du père symbolique (Romain Rolland) de la langue du réel et de l'imaginaire maternels, comme le suggère Elisabeth Geblesco dans son étude psychanalytique sur la métaphore paternelle chez Istrati? (8) Certes, l'auteur attachait une attention particulière à la réception de ses textes, fait attesté d'ailleurs par plusieurs de ses préfaces. Mais, à notre avis, ce n'est pas seulement la fonction documentaire de ses récits qui le préoccupe, tout comme la présence des mots roumains, mais aussi grecs et turcs, ne s'explique pas uniquement par la connaissance limitée du français. Situé à la frontière séparant l'espace de la culture roumaine, essentiellement orale, de celui de l'écriture dans une langue qui n'est pas la sienne, Istrati transforme cet entre-deux en un espace de création hybride. Ses premiers récits, témoignant davantage de la traduction d'un hypothétique texte roumain, traduction émaillée de mots de sa langue maternelle, reflètent son positionnement culturel, renforcé justement par le tissage de ce troisième espace essentiellement hybride .

Revenons un instant sur la confession d'Istrati concernant la difficulté de traduire ses textes français en roumain, tâche qui, à première vue, aurait dû être beaucoup plus facile que son entreprise initiale. Notons tout d'abord que la réception des récits istratiens par un lecteur roumain bilingue présente un double défi. Les mots roumains parsemés dans les textes français, ainsi que les nombreux commentaires métalinguistiques ou descriptifs indispensables à un lecteur ignorant la tradition culturelle roumaine n'ont pas le même effet d'altérité sur un lecteur familier avec cette tradition. Il va sans dire que si le même lecteur choisit la version roumaine des textes istratiens, il constatera que l'espace de la traduction culturelle s'est considérablement rétréci, n'y trouvant plus sa raison d'être, ce qui explique, en partie, la difficulté d'Istrati de traduire en roumain ses propres textes. Si I'on s'en tient aux observations de Tamara Ceban (2006), la disparition du contraste linguistique présent dans les récits écrits en français sera compensée par la présence d'un grand nombre de figures de rhétorique hautement expressives, ainsi que par l'insertion de locutions et d'expressions chargées de connotations absentes du texte d'origine. Il en résulte que l'enjeu de la traduction culturelle n'est pas du tout négligeable dans le cas des récits conçus dans un entre-deux langagier.

En poussant cette réflexion encore plus loin, qu'en est-il de la réception des textes istratiens traduits dans plus de vingt langues ? Comment s'explique leur réception particulièrement favorable dans des pays aussi éloignés que la Turquie et le Japon ? Nous avons des raisons de croire que les réponses à ces questions pourraient nuancer davantage la problématique de la traduction culturelle. Comme il n'est pas de notre dessein d'avancer d'autres hypothèses, nous nous contenterons de signaler que la réédition des œuvres d'Istrati aux éditions Phébus (2006), sous la direction de Linda Lê, atteste un renouveau d'intérêt pour cet auteur qui, dès les années 20, avait compris le rôle capital de la tolérance, de 
I'ouverture vers l'Autre, du multiculturalisme. C'est justement cette thématique chère aux auteurs francophones qui constitue l'objet de l'article «Identités et cultures balkano-méditerranéennes dans l'œuvre de Panaït Istrati », paru dans Francophonie et multiculturalisme dans les Balkans (Steiciuc 2006). Selon l'auteure de I'article, le périple des héros istratiens à travers différentes aires méditerranéennes leur permet de constater la richesse culturelle de chaque peuple, mais aussi la peur de la différence, dont on ne guérit qu'en faisant un effort de comprendre l'Autre, de forger son identité sans exclure l'altérité.

Tous ces messages d'Istrati, plus actuels que jamais, s'expriment à partir de ce troisième espace culturel, espace hybride, dynamique, traversé de tensions identitaires multiples. À notre avis, loin de constituer une trahison par rapport à la langue et à la tradition culturelle roumaines, son choix du français comme langue d'écriture témoigne d'une prise de conscience douloureuse, à savoir la nécessité de s'exprimer (au risque de nombreuses frustrations) dans une langue qui permette à un public plus large d'avoir accès à ses récits. Mais comme les récits d'Istrati tirent leur sève de la tradition roumaine, contaminée par celle balkanique, l'auteur franchit souvent le seuil qui sépare les deux langues. Les mots roumains, turcs, grecs circulent librement dans cet espace frontalier hybride, qui se rétrécira considérablement dans les romans réalistes qu'il écrira vers les années 30 .

\section{Patrick Chamoiseau}

Considérons maintenant le cas de Patrick Chamoiseau. Avant d'aborder la question de l'énonciation culturelle dans son roman bien connu Solibo Magnifique, une précision s'impose : la nouvelle ${ }^{8}$ littérature antillaise ne semble pas répondre à l'horizon d'attente d'un large public local, mais plutôt à celui d'un cercle restreint d'intellectuels. Par contre, la réception critique de cette même littérature, qu'il s'agisse de la réception nord-américaine, européenne ou africaine, s'avère d'une grande richesse, fait attesté, entre autres, par la présence d'un nombre considérable de romans antillais dans les programmes universitaires nord-américains ${ }^{9}$. Il s'ensuit que les auteurs antillais, comme tant d'autres auteurs francophones, pour se faire connaître d'un public plus large, doivent nécessairement choisir le français comme langue d'écriture. Quant à Patrick Chamoiseau, notons dès le début qu'à la différence d'Istrati, il maîtrise parfaitement sa plume française tout en jouant à merveille de la « flûte » créole. Ce n'est donc pas étonnant qu'il figure parmi les récipiendaires du prestigieux prix Goncourt, attribué en 1992 pour son roman Texaco.

http://www.brocku.ca/cfra/voixplurielles05-02/index.html 


\section{Solibo Magnifique}

Le lecteur de Solibo Magnifique, roman publié en 1988, est invité à participer au tissage d'un véritable espace identitaire antillais, suite à la mort subite du conteur Solibo. La figure mythique de Solibo se reconstruit à travers les dépositions, la plupart en créole, de quelques-uns de ses auditeurs, devenus suspects aux yeux des autorités locales ${ }^{10}$. Par le biais d'une intrigue policière construite autour de la mort énigmatique du conteur Solibo, Chamoiseau témoigne de I'agonie du monde de l'oralité, agonie suggérée métaphoriquement par « l'égorgette de la parole » $(25)^{11}$ du conteur.

Conscient de la distance qui sépare le « monde finissant » (227) de l'oral de l'hégémonie incontestable de l'écrit, le narrateur-personnage du roman de Chamoiseau assume quand même la tâche difficile de capter la parole fuyante du conteur et de l'inscrire dans un texte figé dont la logique d'écriture est fournie par celle de l'oral. Mais, avant de mettre sur papier «L'écrit du souvenir », produit de la mémoire collective des auditeurs de Solibo, le narrateur nous offre « L'écrit du malheur » au cours duquel il dresse le portrait complexe de Solibo dans le contexte d'un monde postcolonial en voie de transition vers de nouvelles structures sociales et culturelles. C'est un récit oralisant aux débordements carnavalesques ${ }^{12}$, dont Chamoiseau dévoile la construction au fur et à mesure qu'il raconte les événements.

\section{Entre l'oral et l'écrit}

Fasciné par le verbe inépuisable de Solibo, le marqueur de paroles prend des notes, fait des enregistrements, se mêle parmi les auditeurs du vieux conteur, devenus de plus en plus rares, dans l'espoir de saisir sa parole chargée des pulsions de la vie. De son côté, malgré son scepticisme à l'égard de la possibilité d'écrire la parole, (« On n'écrit jamais la parole, mais des mots » 53), Solibo admire chez son jeune interlocuteur la passion des mots et sa persévérance de « capturer la parole à l'écriture » (52). Bien qu'il sente depuis quelque temps la réduction progressive de l'espace de l'oral en faveur de celui de l'écrit, Solibo entrevoit pourtant la possibilité de donner la main au futur écrivain « par-dessus la distance » (53) qui les sépare : « Je parle, mais toi tu restes. Je parlais, mais toi tu écris, en annonçant que tu viens de la parole » (53), dit-il à Chamoiseau.

L'entreprise ethnographique du marqueur de paroles prendra une direction différente après la mort énigmatique du conteur. Chamoiseau, qui figurait parmi les auditeurs de Solibo, devient tour à tour témoin et suspect dans un crime que le brigadier-chef Bouafesse et plus tard I'inspecteur principal Evariste Pilon veulent prouver à tout prix. Au cours de cette enquête policière tragi-comique où s'affrontent deux mondes qui n'ont rien en commun, ni même la langue, le personnage Chamoiseau constate à quel point ces deux mondes sont incompatibles : « Nous sommes ici dans un frottement de mondes, inspectère, un espace d'érosion, d'effacement où...» (170) À travers la multitude des perspectives tissées par les dépositions des suspects, la plupart en créole, on reconstruit la figure mythique du conteur qui continue à vivre dans la mémoire de ses auditeurs par la force de sa voix et par la qualité de sa performance :

[...] la parole du conteur, c'est le son de sa gorge, mais c'est aussi sa sueur, les roulades de ses yeux, son ventre, les dessins de ses mains, son odeur, celle de sa compagnie, le son du ka et tous les silences. Il faut y ajouter la 
nuit autour, la pluie s'il pleut, les vibrations silencieuses du monde. (47-48)

Cette esquisse des éléments constitutifs des performances de Solibo met en lumière les conditions pragmatiques de toute énonciation orale traditionnelle, dont la complexité est difficile à transposer dans le texte écrit. Toutefois, la difficulté de cette tâche ne décourage pas le marqueur de paroles qui, après la mort du conteur, se donne comme projet de sauver tout ce qu'il peut de la richesse de son dit.

\section{L'écriture de I'entre-deux}

La problématique de la transposition de l'imaginaire créole en français, qui continue à préoccuper les Créolistes, se trouve donc au cœur de ce récit postmoderne. Chamoiseau exprime d'ailleurs sa position au sujet de la possibilité de traduire en français la parole du conteur créole dans de nombreuses réflexions métalinguistiques insérées dans son roman. De surcroît, son inscription dans le texte en tant que personnage lui permet de définir plus clairement son rôle de marqueur de paroles. À l'instar d'Istrati, Chamoiseau refuse l'étiquette d'écrivain et, en interposant entre lui et le conteur la figure du scripteur au nom d'oiseau, il assigne à celui-ci le projet de transformer le dit de Solibo tout en gardant les traces de la diction originelle du paroleur ${ }^{13}$. Par le biais d'une intrigue policière contaminée, elle aussi, par le modèle de la narration orale ${ }^{14}$, il suggère non seulement le danger de la disparition du monde de l'oralité illustrée de façon symbolique par la mort de Solibo, mais aussi la possibilité d'adopter un nouveau traitement générique survenu déjà sous la plume des écrivains antillais. À ce sujet, voici un extrait d'un entretien avec l'auteur de Solibo Magnifique : « La problématique dans laquelle je me situe n'est pas de refaire de l'oral dans l'écriture, d'oraliser l'écrit mais vraiment de trouver un point d'incandescence entre l'oralité et l'écriture [...]. L'écriture doit être aujourd'hui riche de l'oraliture et de l'écriture. » (Plumecock 126)

Ce projet d'écriture particulier s'énonce dès l'épigraphe du roman : « Je suis d'un pays où se fait le passage d'une littérature orale traditionnelle contrainte, à une littérature écrite, non traditionnelle, tout aussi contrainte. Mon langage tente de se construire à la limite de l'écrire et du parler ... » Comme le montre très bien Cheikh M. Ndiaye, l'auteur martiniquais se sert de cette épigraphe pour attirer l'attention à son lecteur sur le fait que « la frontière entre l"écrire" et le "parler" est plus virtuelle que réelle » (119). Autrement dit, le passage de l'oral à l'écrit se fait par l'intermédiaire d'« une écriture fugitive » (119), reflétant une créativité multiforme qui s'insère dans un entre-deux traversé de nombreuses tensions individuelles et collectives.

\section{L'entre-deux de l'écriture}

L'entre-deux de l'écriture de Chamoiseau se définit aussi comme un espace de contact entre le texte et le métatexte, témoignant de la difficulté du choix du discours approprié à son projet scriptural. Son récit hybride à référent antillais, écrit dans la langue de I'Autre pour un public d'Ailleurs, est ponctué par de brefs appels aux auditeurs-lecteurs, appels présents également dans le hors-texte. Tout comme les premiers récits d'Istrati, Solibo Magnifique met en place un contrat de lecture particulier qui programme le lecteur vers l'écoute, en lui mettant sous les yeux un texte écrit fragmenté et 
hétérogène. Afin de capter la complexité culturelle contenue dans la parole du conteur, Chamoiseau émaille son texte de nombreux mots et phrases créoles, ce qui atteste non seulement l'état de diglossie des Antillais ${ }^{15}$, mais aussi un projet d'écriture particulier mettant à I'honneur l'oraliture ${ }^{16}$ qui, de I'avis des Créolistes, devrait inspirer tout récit antillais. En tant que lieu de rencontre et d'interférence d'éléments culturels hétérogènes, où les voix se superposent en créant un véritable chant polyphonique, I'oraliture s'impose aussi comme signe d'une culture polymorphe produite par un sujet fragmenté mais dynamique, parti à la recherche d'un espace culturel qu'il cherche à s'approprier sans effacer les différences.

Notons en passant que quelques années plus tard, dans l'Eloge de la Créolité, véritable défense et illustration d'une littérature spécifiquement antillaise, Chamoiseau, Confiant et Bernabé exprimeront d'une façon explicite l'urgence de mettre en place une esthétique caribéenne inspirée de l'oraliture. La même idée se retrouve chez Edouard Glissant qui propose de placer I'oral « à l'intérieur de l'écriture » (16). On y reconnaît sans doute la solution du scripteur de Solibo, déterminé à faire l'apprentissage d'une nouvelle écriture, dictée par les conditions postcoloniales des Antilles qui mettent en danger les anciennes traditions orales. À ce sujet, il suffit de relire les commentaires finals du personnage Chamoiseau qui se demande à juste titre comment reconstituer le dernier dit de Solibo sans trahir le texte oral ${ }^{17}$. Aidé par quelques « survivants » (226) qui avaient assisté à la dernière performance du conteur, il extrait une version abrégée et appauvrie des mots du Magnifique en guise de témoignage d'un monde qu'il refuse de laisser mourir. Entre « L'écrit du malheur » d'avant la parole et « L'écrit du souvenir » d'après la parole le narrateur tisse un texte à l'aspect protéiforme, qui n'est autre que le tiers espace de l'énonciation culturelle, espace frontalier capable de suggérer la richesse d'une culture hybride. Cette transposition du dit de Solibo porte encore la trace de la parole foisonnante du conteur, dont la force performative se fait sentir à travers la page écrite. Delphine Perret souligne à juste titre la tendance vers l'excès manifestée par le narrateur de Solibo Magnifique : « excès de vie, excès imaginatif, excès aussi dans la manipulation d'autrui » (829). Mais en même temps, Chamoiseau privilégie « l'esthétique de l'inachevé ou du suspens » (Chancé $2003: 870$ ), ajoutant également une dimension ludique à son texte. Il a sans doute réussi à incorporer la créolité de l'expression orale dans le français de la transcription, tout comme Istrati avait incorporé la roumanité de son imaginaire dans la langue de son père symbolique. Dans les deux cas, même s'il s'agit d'un pseudo-acte de langage, d'une performance fictive qui ne saurait capter les innombrables facettes de la performance du conteur créole ou roumain, la représentation de sa parole refait partiellement I'acte performatif originaire, où le dire acquiert la force du faire. Citons de nouveau Dominique Chancé, qui met en évidence l'importance de l'entreprise de Chamoiseau, justifiée principalement par l'urgence d'empêcher la disparition du monde de la parole, condamné par la civilisation de l'écrit :

Dans Solibo Magnifique, le narrateur précisément ne croit plus qu'il puisse imiter, prolonger tout simplement le conteur. Il entre en dialogue avec le conteur Solibo, pour dire son échec, son incapacité à entrer dans une filiation directe au conteur. Si écrire, en effet, c'est trahir la parole, l'enjeu du récit et du texte, dans son énonciation, devient l'invention d'un dispositif qui permette d'écrire tout de même la parole ». (2003: 874)

Pour que l'écriture ne fige pas complètement la parole, le personnage Chamoiseau recourt à plusieurs techniques, déjà analysées par ses critiques, dont nous nous contentons d'en rappeler deux : la suppression de la ponctuation afin de suggérer la fluidité de la parole et le recourt à une variété de procédés sonores et graphiques indiquant l'insertion de l'oral 
dans l'écrit (Bérard 99). Tout comme son confrère Istrati, I'écrivain Chamoiseau laisse entendre dans son texte une pluralité de voix capables d'être réactivées lors de l'acte de lecture, une lecture déjà programmée par de nombreux éléments textuels.

\section{Conclusion}

Pour conclure, une dernière précision : le projet de transposer l'oral à l'écrit dans les conditions où la langue d'écriture (en l'occurrence le français) est différente de celle de la diction originelle (le roumain ou le créole) pose un double défi à des écrivains comme Istrati et Chamoiseau. Si I'on accepte, par exemple, que I'oraliture que Chamoiseau veut capter dans son roman n'est autre chose que « dire en créole et écrire en français » (Figueiredo 31), on pourrait conclure, par extrapolation, que l'écriture de tout écrivain francophone soucieux de transposer une énonciation culturelle particulière sera infusée, à des degrés variables, par sa langue maternelle. Dans le cas de nos deux auteurs, on constate facilement à quel point les niveaux lexical et syntaxique sont touchés par leur langue d'origine. Ces considérations nous ramènent au point de départ : s'agit-il d'une trahison du dire créole ou roumain, ou plutôt d'une traduction doublement enrichissante, exigée par le contact de deux langues et de deux cultures ? $^{18}$ Nous espérons avoir démontré que l'hypothèse de la traduction, génératrice d'un espace d'écriture frontalier jouit de plus d'arguments, fait appuyé, d'ailleurs, par la réception favorable des auteurs issus de milieux culturels très divers. Ce n'est donc pas étonnant qu'un autodidacte comme Istrati travaille cet espace dans tous les sens, car il représente un lieu privilégié pour l'énonciation de son riche bagage culturel, et cela bien avant que les termes d'identité, d'altérité, de multiculturalisme ne deviennent des concepts largement véhiculés dans les études littéraires. D’autres écrivains, comme Chamoiseau, se servent de ce troisième espace pour transposer dans leurs textes des situations interculturelles particulières, liées à différentes aires géographiques. Cette pratique d'écriture, intimement liée à l'intertextualité, faisant souvent appel au collage d'éléments non textuels (la voix, l'image), leur permet de mieux aborder la question de la relation entre une identité culturelle de plus en plus métissée et nomade et la pluralité des langues du village global.

http://www.brocku.ca/cfra/voixplurielles05-02/index.html 


\section{Références}

Bhabha, Homi. The Location of Culture . London et New York : Routledge, 1994.

Bălan, Zamfir. Panait Istrati. Tipologie narativă [Panaït Istrati. Typologie narrative]. Braïla : Istros, 2001.

Bérard, Stéphanie. «Patrick Chamoiseau, héritier du conteur ? Respect ou trahison de la tradition orale dans Manma Dlo contre la fée Carabosse ». Langue et identité narrative dans les littératures de l'ailleurs : Antilles, Réunion, Québec. Aix-en-Provence : Université de Provence, 2005 : 91-105.

Bernabé, Jean, Patrick Chamoiseau, Raphaël Confiant. Eloge de la créolité. Paris : Gallimard, 1989.

Ceban, Tamara. « Eléments orientaux dans l'écriture de Panaït Istrati ». Conférence présentée dans le cadre du Colloque international La Francophonie comme vecteur de la communication. ULIM, Chişinău, mars 2006.

Chamoiseau, Patrick. Solibo Magnifique. Paris : Gallimard, 1988.

Chamoiseau, Patrick et Raphaël Confiant. Lettres créoles. Tracées antillaises et continentales de la littérature. Paris : Hatier, 1991.

Chancé, Dominique. L'Auteur en souffrance. Essai sur la position et la représentation de l'auteur dans le roman antillais contemporain (1981-1992). Paris : Presses Universitaires de France, 2000. . «De Chronique des sept misères à Biblique des derniers gestes, Patrick Chamoiseau est-il baroque ? ». MLN 118. 4 (2003) : 867-894.

Condé, Maryse. Traversée de la mangrove. Paris : Mercure de France, 1989.

Condei, Cecilia. Interferenţe lingvistice romano-franceze în opera lui Panait Istrati [Interférences linguistiques entre le roumain et le français dans l'œuvre de Panaït Istrati]. Braïla : Istros, 2001.

Covaci, Vasile. « Expressions et locutions roumaines dans les écrits de Panaït Istrati ». Cahiers Roumains d'Études Littéraires 1 (1981) : 54-63. . «L'Guvre de Panaït Istrati ou le problème de traduire une civilisation ». in Revue Roumaine de Linguistique 16 (1979) : 193-98.

Figueiredo, Euridice. «La réécriture de I’histoire dans les romans de Patrick Chamoiseau et Silviano Santiago ». Études littéraires 25.3 (Hiver 1992-1993) : 27-38.

Geblesco, Elisabeth. Panaït Istrati et la métaphore paternelle. Paris : Anthropos, 1989.

Glissant, Edouard. « Le Chaos-monde, I'oral et l'écrit ». In Ralph Ludwig (dir.), in Écrire la parole de la nuit. La nouvelle littérature antillaise. Paris : Gallimard 1994 : 111-29.

Hormière, Jean. «Adrien Zograffi, roman de formation », in Panaït Istrati, notre contemporain. Le livre du centenaire 1884-1984. Aix-en-Provence : Édisud, 1986 : 57-64.

Ionescu, Mariana. Les (En)jeux de l'oral et de l'écrit : le cas de Panaït Istrati. Braïla : Istros, 2000.

Istrati, Panaït. « Hors du monde, dans le monde ». Esprit 3, 1977 [1921] : 375-81.

http://www.brocku.ca/cfra/voixplurielles05-02/index.html 
Kyra Kyralina. Paris : Gallimard, 1985 [1924].

. Fuvres de Panaït Istrati 1. Paris : Gallimard, 1968.

. Présentation des haïdoucs. Paris : Gallimard, 1983 [1925].

Jutrin-Klener, Monique. Panaït Istrati, un chardon déraciné : écrivain français, conteur roumain. Paris : Librairie François Maspero, 1970.

Laroche, Maximilien. La Double Scène de la représentation : oraliture et littérature dans la Caraïbe. Québec : Université Laval, GRELCA, 1991, coll. essais 8.

Milne, Lorna. Patrick Chamoiseau. Espace d'une écriture antillaise. Amsterdam-New York : Rodopi, 2006.

Moudileno, Lydie. L'Écrivain antillais au miroir de sa littérature : mises en scène et mises en abyme du roman antillais. Paris : Karthala, 1997.

Ndiaye, Cheikh M. « Marronnage, oralité et écriture dans Solibo Magnifique de Patrick Chamoiseau ». Nouvelles Etudes Francophones 22.2 (Aut. 2007) : 112-121.

Nouvelles écritures francophones : vers un nouveau baroque? Jean Cléo Godin (dir.). Montréal : Presses de I'Université de Montréal, 2001, pp. 7-8).

Panait Istrati, notre contemporain. Le livre du centenaire 1884-1984. Aix-en-Provence : Edisud, 1986.

Perret, Delphine. « La Parole du conteur créole : Solibo Magnifique de Patrick Chamoiseau ». The French Review 67.5 (1994) : 824-39.

Plumecock, Michaël. «Entretien avec Patrick Chamoiseau autour de Solibo Magnifique ». Roman 2050.27 (1999) : 125-35.

Schaffner, Alain. « Panaït Istrati, ou la conquête de la langue ». Esprit créateur 44. 2 (Été 2004) : 51-60.

Sourieau, Marie-Agnès. «Patrick Chamoiseau, Solibo Magnifique From the Escheat of Speech to the Emergency of Language ». Callaloo 15.1 (1992) : 131-137.

Steiciuc, Elena-Brandusa. « Identités et cultures balkano-méditerranéennes dans l'œuvre de Panaït Istrati », in Francophonie et multiculturalisme dans les Balkans. Paris : Éditions Publisud, 2006 : $69-81$.

Wells, Catherine. L'oraliture dans Solibo Magnifique. Québec : Université Laval, GRELCA, 1994, coll. essais 12. 
${ }^{1}$ Chamoiseau préfère cette appellation à celle d'écrivain $(1988,44)$. Il est à remarquer qu'Istrati, se servant de l'instance préfacielle, rappelle lui-aussi à ses lecteurs qu'il est beaucoup plus proche du conteur que de l'écrivain.

2 À partir du XVII ${ }^{\mathrm{e}}$ siècle, le mot haïdouc désignera les hors-la-loi qui s'érigent en défenseurs des opprimés et combattent contre l'oppression interne et externe.

3 Dans son article «Adrien Zograffi roman de formation » (1986), Jean Hormière propose de lire ce cycle romanesque comme une série de récits marquant la formation du jeune protagoniste. Au début, Adrien accomplit sa formation en écoutant les histoires des autres; plus tard, à travers les nombreux voyages qu'il entreprend, seul ou en compagnie de quelques amis fidèles.

${ }^{4}$ Dans Panaït Istrati et la métaphore paternelle, Elisabeth Geblesco appelle ces quatre récits « le cycle de Cosma », d'après le nom du haïdouc présent dans ces premiers textes d'Istrati (6).

${ }^{5}$ « Cu Panaït Istrati despre : viata, oameni, destinul meu,...». Entretien accordée à G. MihairZamfirescu, publiée dans Romînia Literară, mai 1933 (cité par Jutrin-Klener 1970, 260).

${ }^{6}$ Pour de plus amples renseignements à ce sujet, voir mon livre Les (En)jeux de l'oral et de l'écrit : le cas de Panaït Istrati, 2000.

7 Les détails concernant les interférences linguistiques chez Istrati abondent dans les études de Vasile Covaci $(1979,1981)$ et de Cecilia Condei (2001).

${ }^{8}$ Cette épithète décrit l'état d'émergence d'une littérature moderne qui tire sa sève d'une riche tradition orale.

9 La question de la réception de la littérature antillaise a constitué l'objet d'un article antérieur publié dans Dialogues Francophones 8-9 (2003) : 257-267)

10 De même que les personnages de Traversée de la mangrove de Maryse Condé, qui se remémorent les circonstances de leur rencontre avec le mystérieux Francis Sancher, les auditeurs de Solibo fouillent dans leurs souvenirs afin de reconstituer fidèlement la figure du Magnifique. Mais, à la différence des souvenirs des habitants de Rivière au Sel, dominés en grande partie par la haine et les inimitiés, ceux des fidèles de Solibo nous permettent d'esquisser le portrait d'une figure mythique qui n'a rien perdu de la dignité du conteur d'autrefois, en dépit des nouvelles conditions, autrement aliénantes, des structures antillaises postcoloniales.

11 Il s'agit d'un curieux étranglement « de l'intérieur »(201), comme le constate le médecin qui avait performé I'autopsie de Solibo. Si ce diagnostic rend perplexes les enquêteurs du soi-disant meurtre, il est parfaitement compréhensible dans une communauté qui voit l'espace de l'oral se rétrécir rapidement sous les coups de l'écrit. Mourir étranglé par sa propre parole renvoie métaphoriquement à la disparition inévitable d'une civilisation bâtie sur la force de la parole vive des conteurs.

12 On parle de plus en plus souvent d'une « écriture baroque », propre aux nouvelles littératures francophones qui valorisent «I'insolite et l'incongru [...], I'hétérogène et le métissage » (Nouvelles écritures francophones : vers un nouveau baroque?, 2001, 7-8).

13 Tout comme Istrati, Chamoiseau n'échappe lui non plus à une lecture psychanalytique. Si le premier avait été associé à Romain Rolland, considéré père symbolique d'Istrati par E. Geblesco, le Martiniquais est associé soit à Aimé Césaire (Lydie Moudileno), soit au conteur (D. Chancé 2000), deux pères spirituels de l'écrivain qui n'ose se projeter dans son texte qu'en tant que « marqueur de paroles ». Lorna Milne reprend cette discussion dans son livre consacré à Patrick Chamoiseau.

${ }^{14}$ Dans son étude consacrée à Solibo Magnifique, Catherine Wells remarque à juste titre que «la manière dont le narrateur arrange des segments de son histoire rappelle la figure de l'ordre dans le roman policier non pas pour valoriser une figure d'autorité, comme le ferait la forme littéraire moderne, mais plutôt pour l'attaquer discrètement et implicitement comme le faisait le conteur » (159). D'ailleurs, le titre du roman de Chamoiseau suggère au lecteur qu'il s'agit d'une célébration de la victime et non pas de l'inspecteur.

${ }^{15}$ Voir à ce sujet l'article de Marie-Agnès Sourieau.

${ }^{16}$ Dans l'ouvrage Lettres créoles, l'oraliture est définie comme le produit de plusieurs «tracées», « infinies petites sentes » qui « témoignent d'une spirale collective que le plan colonial n'avait pas prévu » (12). 
17 Dès le début de sa carrière Patrick Chamoiseau choisit de mettre le conteur et sa parole au centre de sa première pièce de théâtre, intitulée Manman Dlo contre la fée Carabosse. À ce sujet, Stéphanie Bérard se propose de répondre à la question suivante : en tant qu'héritier du conteur, l'écrivain Chamoiseau continue-t-il la tradition orale ou la trahit suite au passage à l'écriture? En extrapolant la conclusion de Bérard, nous dirons que le « marqueur de parole » de Solibo Magnifique, tout comme le conteur qui subit un procès dans la pièce de théâtre de Chamoiseau, choisit d'« infuser la parole dans l'écriture » (Bérard 95).

${ }^{18}$ En se référant aux écrivains antillais, Euridice Figueiredo constate que dans le cas de la créolisation du français ces écrivains ont fait appel à « une double traduction qui change forcément le rythme de la phrase, la valeur sémantique des mots, bref, toute une vision du monde implicite dans une langue. » (31) 